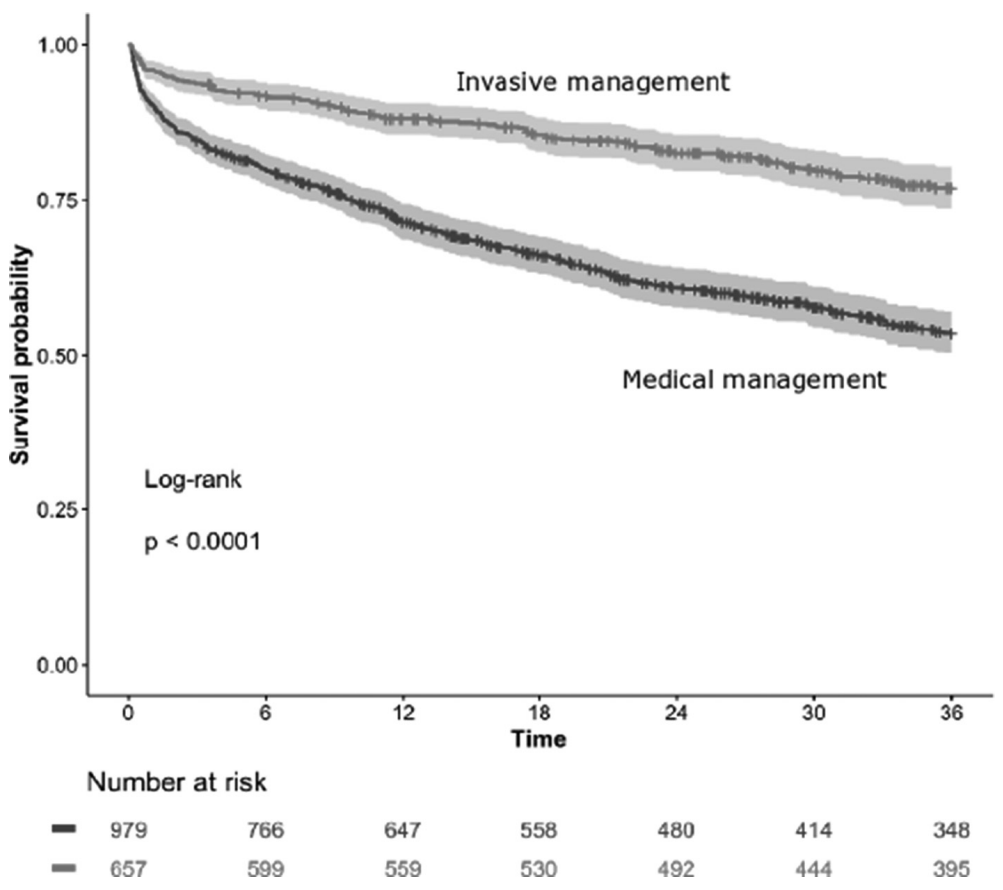

Abstract 57 Figure 1 Cumulative survival in the invasive and medical management groups

association if there is an unmeasured covariate with a relative risk of at least 2.61 for both mortality and undergoing invasive management. The highest mortality HR for comorbidities included in our model were heart failure (HR 2.06 (95\% CI 1.60-2.63)) and aortic stenosis (HR 1.95 (95\% CI $1.42-$ 2.67)).

Conclusion This study provides evidence that the survival advantage from invasive management may extend to elderly patients with NSTEMI. Future research should address the possibility of unmeasured confounding, including by postadmission prognostic factors that affect choice of invasive or medical management.

Conflict of Interest No conflict of interest

\section{$58 \quad$ BI-RADIAL VERSUS FEMORAL ACCESS IN CHRONIC TOTAL OCCLUSION REVASCULARISATION}

Mohammed Meah*, Wern Yew Ding, Jonathan Hasleton, Nick Palmer. NHS

\subsection{6/heartjnl-2019-BCS.56}

Aim Revascularisation of CTOs is a technically challenging procedure and complex cases often require dual access. Advancements in techniques have made bi-radial access more feasible. However, the limited evidence suggests that in patients with complex lesions radial access is associated with lower success rates. Our primary outcome was to determine how effective bi-radial access is compared to femoral.

Methods This was a retrospective single centre observational study. Patients who had undergone CTO-PCI between January 2014 and January 2018, that required dual-access, were enrolled. They were separated into bi-radial access and radiofemoral or bi-femoral access. Every angiogram was reviewed independently to calculate a JCTO score (a measure of lesion complexity). Data on demographics, co-morbidities, complications, lesion characteristics, radiation and contrast dose were collected. Standard univariate analyses were performed to look for any tends that predicted failure to revascularise.
Results In total, 150 cases were identified, 109 bi-radial, 41 femoral (36 radio-femoral and 5 bi-femoral) access. Although there was no statistically significant difference in success rate for revascularisation between the radial and femoral groups $(87 \%$ vs $78 \% ; \mathrm{p}=0.17)$, there was a trend in favour of radial access. The average JCTO score was 3 vs $4 \quad(p=0.04)$ indicating that those undergoing femoral procedures had more complex lesions. When only comparing cases with a JCTO score of 4 , success rates were equivalent $(78.1 \%$ vs $80.9 \% \mathrm{p}$ $=>0.99$ ).

Although statistical significance was not reached due to the overall small population, the following were associated with CTO-PCI failure: raised BMI, poor renal function, previous CABG, a higher JCTO score, CTO length $>20 \mathrm{~mm}$, presence of a $>45^{\circ}$ bend within the diseased segment and the absence of collateral vessels. The radial access group had shorter procedure lengths (111 minutes (68-137) vs 147 minutes (100$165) ; \mathrm{p}=0.02$ ), reduced radiation exposure (dose area product $17452 \mathrm{CGYcm}_{2}(9827-23958)$ vs $23651 \mathrm{CGYcm}_{2}$ (1530327016); $\mathrm{p}=0.05)$ and required less contrast $(237 \mathrm{mls}(180$ $320)$ vs $315 \mathrm{mls}(256-345) ; \mathrm{p}=0.04)$. There was also a reduction in mean length of in-hospital stay $(0.38 \pm 1.3$ vs $0.61 \pm 1.1$ days $[\mathrm{p}=0.02])$. A trend towards a higher rate of complications was noted in the femoral group $[10.1 \%$ vs $19.5 \% \mathrm{p}=0.12$ ] (table 1 and table 2).

Conclusion Bi-radial access is a safe alternative to radio-femoral or bi-femoral access in CTO-PCI. Previous studies have suggested that more complex cases require femoral access. However, when only JCTO 4 patients were compared there was equivalent rates of success. We use 7 French sheaths routinely in bi-radial access. This provides more support and may partly explain why success rates are comparable. With shorter length of stay, fewer complications, and less contrast/ radiation used in radial cases we suggest that even in complex CTO-PCI cases, bi-radial access is an effective and safer alternative.

Conflict of Interest None 